\title{
Archéopages
}

Archéopages

Archéologie et société

34 | 07/2012

Campagnes

\section{La maquette numérique issue de levés 3D}

\section{Sylvie Eusèbe}

\section{OpenEdition}

\section{Journals}

Édition électronique

URL : https://journals.openedition.org/archeopages/426

DOI : 10.4000/archeopages.426

ISSN : 2269-9872

\section{Éditeur}

INRAP - Institut national de recherches archéologiques préventives

\section{Édition imprimée}

Date de publication : 1 février 2012

Pagination : 104-105

ISSN : 1622-8545

\section{Référence électronique}

Sylvie Eusèbe, "La maquette numérique issue de levés 3D », Archéopages [En ligne], 34 | 07/2012, mis en ligne le 01 juillet 2012, consulté le 11 février 2022. URL : http://journals.openedition.org/ archeopages/426 ; DOI : https://doi.org/10.4000/archeopages.426 
œuvre d'un relevé en $3 \mathrm{D}^{\mathbf{1}}$. Des relevés de ce type avaient été précédemment réalisés en région Centre, au cours des travaux préalables à la construction de l'autoroute A19, pour une structure similaire découverte sur le site de Villerau « Le Marjolet » (Loiret), (Gilotte et al., 2008, p. 272-317, fig. 126-128). Cependant, le rendu général en « fil de fer », les relevés manuels et les restitutions produites nous paraissaient insuffisants pour appréhender l'organisation générale de la structure et rendre compte des volumes des différentes galeries et cavités. Compte tenu du laps de temps relativement réduit dédié à cette partie de lintervention (dix jours ouvrés), nous avons opté pour une méthode de relevé plus rapide et plus complète en faisant appel à la société de géomètres experts « Axis conseil ».

La fouille et le relevé classique des stratigraphies et des profils des principales cavités et galeries de ce souterrain ont été réalisés en sept jours. À l'issue de cette première phase d'intervention, trois journées ont été nécessaires pour la réalisation du relevé des contours du souterrain à l'aide d'un émetteur laser permettant de modéliser les volumes et de procéder à une photogrammétrie ${ }^{2}$ et quelques jours supplémentaires ont permis l'exploitation des données et l'élaboration des plans, coupes et profils.

Le premier essai de modélisation a consisté en une tentative de texturation numérique (morphing), qui n'était pas satisfaisante car trop illisible [ill. 1-2]. Puis nous ont été fournies des vues à $360^{\circ}$ des différentes composantes du souterrain, intéressantes malgré quelques déformations, mais qui restent difficiles à exploiter [ill. 3]. La dernière étape a été un mélange entre traitement $3 \mathrm{D}$ et photogrammétrie, avec l'application des prises de vue dans la modélisation $3 \mathrm{D}$ [ill. 4].

Les apports de ce type de relevé peuvent être exposés en quelques points. Le premier est la rapidité d'acquisition des données car, pour ce type de structure, le recours à un relevé classique s'avère difficile à réaliser sans procéder à l'ouverture des voûtes. Ces terrassements, outre le fait qu'ils entrainent la destruction d'une grande partie du terrain sondé, peuvent également fragiliser la construction et entrainer la condamnation de tout ou partie de l'ouvrage. Deuxièmement, la précision des prises de points réduit la marge d'erreur par rapport à un traitement manuel, en particulier dans les endroits difficiles d'accès (pour traiter par exemple des traces d'outils). Troisièmement, la géolocalisation du relevé $3 \mathrm{D}$ annule les divers problèmes d'orientation inhérents à toutes les structures souterraines. L'ultime argument en faveur de cette méthode est la facilité de manipulation et d'interprétation de la structure. Il a suffi de demander une vue verticale générale et de choisir les différents profils voulus, en particulier le profil du développé général du souterrain [ill. 5].

Le recours à la restitution en $3 \mathrm{D}$ pour le souterrain d'Ormes permet un certain nombre de réflexions autour du traitement des cavités souterraines. Une étude préliminaire apparaît indispensable tant du point de vue de la sécurité des différents intervenants, que du point de vue logistique. Cela permet en effet une organisation de la fouille visant à favoriser la mise en place d'une méthodologie associée à l'emploi de ce type de technologie. Contrairement à l'idée encore trop répandue dans la communauté archéologique, la modélisation en $3 \mathrm{D}$ ne sert pas uniquement à la valorisation des vestiges ${ }^{3}$ : elle permet également un enregistrement rapide et précis, et offre une possibilité de traitement a posteriori de l'ensemble des données acquises.

\section{La maquette numérique issue de levés $3 \mathrm{D}$}

Sylvie Eusèbe, Inrap

Le relevé total de vestiges semble désormais accessible grâce à la maquette virtuelle en $3 \mathrm{D}$, issue de la photogrammétrie numérique ou du laser-scanner, procédés qui captent mécaniquement la réalité dans sa géométrie et ses couleurs. Considérée comme un summum de justesse, d'exhaustivité et d'objectivité, cette maquette numérique est souvent présentée comme le support idéal, quelles que soient les études envisagées, à la différence d'un relevé traditionnel spécifique au secteur de l'étude à laquelle il participe. Il convient néanmoins de ne pas oublier qu'un modèle numérique est dit objectif en raison de l'absence d'observation et d'interprétation directement associées, et qu'il ne constitue pas, à lui seul, un « relevé archéologique».

En archéologie préventive, ces technologies mises en œuvre dans un cadre expérimental devraient permettre, entre autres, de tester les différentes méthodes de modélisation et la qualité (précision, résolution, etc.) des résultats selon des protocoles connus et décrits. Elles devraient surtout nous inviter à nous interroger sur nos besoins actuels et à venir en termes de précision nécessaire et suffisante pour tel ou tel type d'observation, ainsi que, plus généralement, sur l'usage que les archéologues peuvent en faire.

La maquette numérique issue de levés en $3 \mathrm{D}$ renouvelle l'apport des vues perspectives et de la maquette " réelle », depuis longtemps utilisées en archéologie, comme outil d'aide à la recherche et comme support de communication. Cependant, de nouveaux défis devront être relevés pour assurer le succès de ces modèles numériques, notamment: garantir leur pérennité (indexation, archivage, métadonnées, interopérabilité) ; garantir leur qualité scientifique, ou tout au moins la connaitre; permettre leur appropriation par l'archéologue, car les objectifs scientifiques induisent les relevés à réaliser et les moyens à mettre en œuvre. La véritable innovation ne consiste ainsi plus à acquérir les compétences techniques pour réaliser ces modèles, mais plutôt, d'une part, à acquérir la maitrise de l'évolution qualitative des données produites et, d'autre part, à maitriser leur exploitation et leur présentation.
1. Nous renvoyons aux
débats et aux actes des

Journées informatique

sciencesconf.org/..

est une technique qui

permet de reconstitue

à partir d'une série

de photographies prises

selon un protocole adapte.

être envisagée avec la

d'animations vectorielles. 